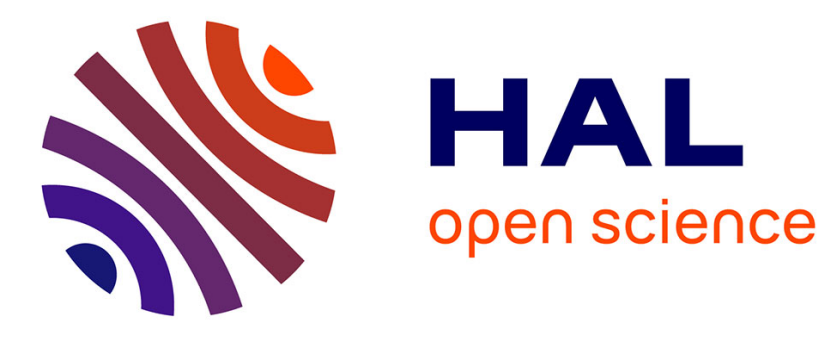

\title{
Vers de nouvelles régions
}

Thérèse Saint-Julien, Denise Pumain, Violette Rey, François Gay, Pierre-Yves

Le Rhun, Maryse Clary

\section{To cite this version:}

Thérèse Saint-Julien, Denise Pumain, Violette Rey, François Gay, Pierre-Yves Le Rhun, et al.. Vers de nouvelles régions. Géographes associés, 1990, 8 (1), pp.35-44. 10.3406/geoas.1990.1730 . hal02885540

\section{HAL Id: hal-02885540 \\ https://hal.science/hal-02885540}

Submitted on 30 Jun 2020

HAL is a multi-disciplinary open access archive for the deposit and dissemination of scientific research documents, whether they are published or not. The documents may come from teaching and research institutions in France or abroad, or from public or private research centers.
L'archive ouverte pluridisciplinaire HAL, est destinée au dépôt et à la diffusion de documents scientifiques de niveau recherche, publiés ou non, émanant des établissements d'enseignement et de recherche français ou étrangers, des laboratoires publics ou privés. 


\section{Vers de nouvelles régions}

Thérèse Saint-Julien, Denise Pumain, Violette Rey, François Gay, Pierre-Yves Le Rhun, Maryse Clary

\section{Citer ce document / Cite this document :}

Saint-Julien Thérèse, Pumain Denise, Rey Violette, Gay François, Le Rhun Pierre-Yves, Clary Maryse. Vers de nouvelles régions. In: Géographes associés n`8,1990. De la France des départements à l'Europe des régions. Géoforum Nantes 89 . pp. 35-44;

doi : https://doi.org/10.3406/geoas.1990.1730

https://www.persee.fr/doc/geoas_1266-4618_1990_num_8_1_1730

Fichier pdf généré le 09/11/2019 


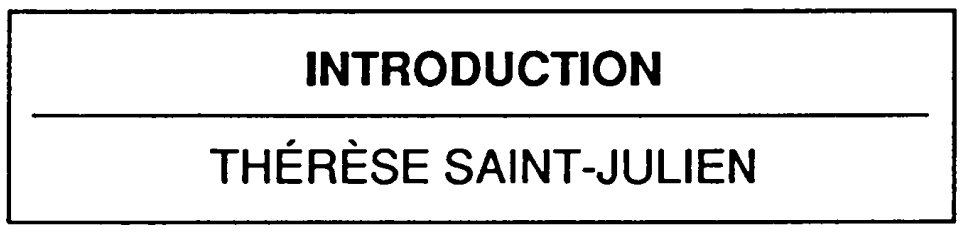

Parler de régions, ou de nouvelles régions, c'est parler de la différence géographique, des échelles de cette différence, des interactions qui l'entretiennent, la réduisent ou la renouvellent.

C'est aussi s'interroger sur le statut de la différence dans les sociétés complexes, et plus précisément de la différence géographique, différence, diversité à valoriser, à produire ou à réduire. Car, en arrière-plan, préside la grande interrogation sur les rapports qu'entretiennent justice sociale et inégalité territoriale.

C'est encore définir les dynamiques qui engendrent, aujourd'hui, les différences territoriales. Evoquer l'idée de nouvelles régions, c'est tout à la fois faire l'hypothèse que les 22 régions françaises ne sont pas tout à fait à la dimension de ce que l'on est en droit d'attendre d'elles et que le territoire national continue de fonctionner comme un système suffisamment fermé pour que des formes spécifiques de diversité géographique, de différenciation territoriale, s'y maintiennent, voire s'y recréent ; qu'aux frontières, des discontinuités apparaissent.

Enfin si l'on s'interroge sur de nouvelles régions, il faut être au clair sur l'objectif ancien ou nouveau que l'on prétend leur assigner.

Ces interrogations pourraient guider la discussion ouverte sur de nouvelles régions. On pourrait, puisque la question de nouvelles régions est posée en référence à l'espace européen, donner aussi en introduction quelques répères sur l'amplitude des disparités interrégionales en France, comparée à celle enregistrée dans les autres grands pays européens.

\section{LES CONTRASTES DU TERRITOIRE FRANÇAIS DANS L'ESPACE EUROPÉEN}

Nous avons montré que certains des grands contrastes qui structurent l'espace français appartiennent à l'architecture générale de l'espace européen. On peut néanmoins penser que les territoires nationaux continuent de fonctionner comme des systèmes suffisamment fermés pour que des formes spécifiques de diversité géographique s'y maintiennent, voire s'y recréent, et pour qu'aux frontières des Etats des discontinuités apparaissent. Si l'on continue d'évoquer davantage le morcellement du vieux continent que son intégration territoriale, les parties plus que le tout, cela tient-il aux habitudes de penser et de voir, à la force des héritages, ou à l'intuition que la dynamique de l'espace européen s'alimente du renouvellement permanent des formes de sa diversité ? A l'échelle européenne, le territoire français apparaît-il davantage défini par ses spécificités, que par les gradients extra-nationaux qui le traversent? 


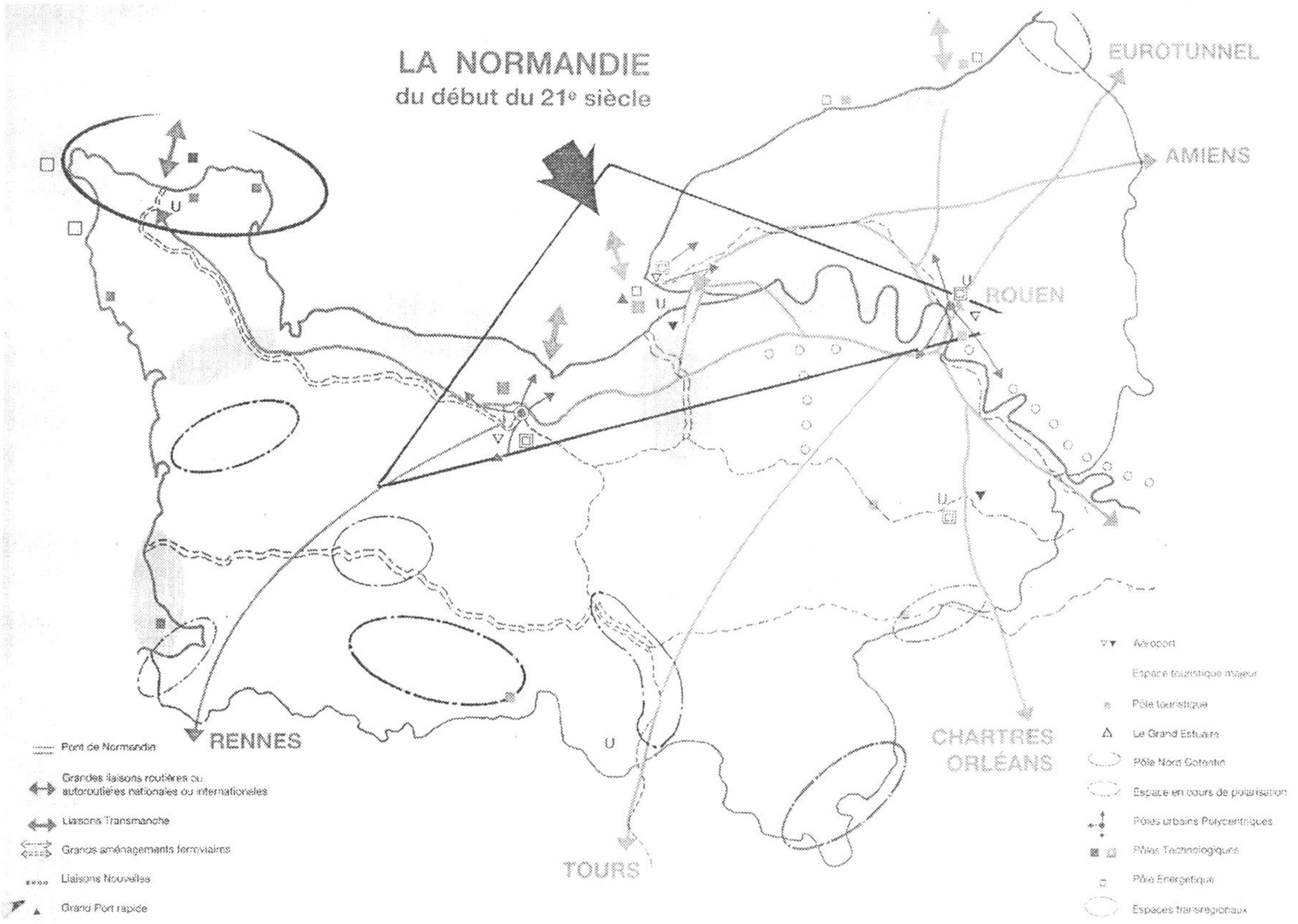




\section{DES DISPARITÉS}

Dans cette comparaison, nous nous référons au moins autant aux concepts de déséquilibre et d'inégalité géographique. L'idée sous-jacente est que le territoire est porteur d'inégalités sociales, ou encore, ce qui peut revenir au même, que des déséquilibres territoriaux trop importants sont un handicap pour le développement du pays. Les Français n'ont-ils pas tendance à penser que, parmi les autres territoires nationaux du Nord-Ouest européen, leur territoire est un de ceux où la distribution géographique de la population et celle des activités sont des plus irrationnelles, où les chances des populations, du fait de leur appartenance régionale, sont parmi les plus inégales ? L'expression "Paris et le désert français", précédemment évoquée, n'a-t-elle pas été pendant près de vingt ans, comme un cri de révolte, l'expression du déséquilibre suprême que la Province se devait de ne plus accepter ? Semblable perception assez communément répandue, trop oublieuse de l'importance des interdépendances des régions, peu sensible sans doute à l'existence des effets positifs d'une croissance, par définition géographiquement déséquilibrée, a-t-elle des fondements aussi solides qu'il y parait?

\section{DES INÉGALITÉS RÉGIONALES MOYENNES POUR L'EUROPE}

La répartition très inégalitaire de la population sur le territoire national, qui est un fait majeur de l'individualisation de la situation française en Europe, a été longuement évoquée. En revanche, quand on considère les disparités démographiques et socio-économiques interrégionales, toutes choses égales quant à la masse démographique des régions, les écarts ne sont en moyenne ni systématiquement plus forts, ni systématiquement plus faibles en France que dans les grands pays voisins. La France apparait en position médiane entre l'Italie, où les différences interrégionales ont tendance à être toujours plus élevées qu'ailleurs, et le Royaume-Uni où les disparités sont en général plus faibles. La position médiane de la France est partagée par l'Allemagne : selon les indicateurs retenus, la moyenne des écarts interrégionaux est plus ou moins élevée que la moyenne française, tout en restant très proche.

Les critères démographiques sont très peu discriminants. Pour un même pays, les situations démographiques régionales sont peu contrastées, et les situations des différents pays sont très voisines; les écarts enregistrés entre les régions françaises sont aussi faibles qu'ailleurs.

La composition de la population active en trois secteurs d'activité crée entre les régions des pays de l'Europe Communautaire des écarts tout à fait comparables. Dans l'en- 
semble, l'importance des spécialisations de l'activité régionale est en France tout à fait moyenne, comparée à celle de l'Allemagne, du Royaume-Uni ou de l'italie.

Quelques indicateurs plus directement révélateurs de l'ampleur des inégalités sociospatiales tels les taux de chómage, le montant du produit intérieur brut par habitant ou de la valeur ajoutée par personne occupée, révèlent qu'en la matière, la situation française est plutót moins inégalitaire que celle des autres pays, le Royaume-Uni excepté.

Si l'on considère que les écarts des régions à la situation moyenne jouent un rôle d'autant plus grand, dans l'expression des déséquilibres géographiques, que les régions ont un poids plus important, on peut apprécier l'importance des disparités régionales en pondérant leur mesure par la taille démographique ou économique des régions. Les conclusions précédentes sont confirmées et tout au plus nuancées. Ainsi appréciés, l'ampleur des déséquilibres interrégionaux français apparaît moyenne, proche de celle enregistrée pour le Royaume-Uni et, le plus souvent, légèrement supérieure à celle notée en Allemagne.

En conclusion, on peut donc affirmer que, contrairement à une idée très répandue en France, les disparités socio-économiques interrégionales y sont loin d'apparaître comme les plus extrêmes, parmi les pays européens occidentaux de taille comparable. Seule la répartition géographique des masses de population fait exception. Cet héritage des forces centralisatrices est contrebalancé par une assez grande homogénéité des populations : la France n'est-elle pas le pays d'Europe où les dialectes et les accents régionaux ont le moins gardé leurs spécificités?

\section{CONTRASTES RÉGIONAUX ET FORCE DES APPARTENANCES NATIONALES}

La seconde question peut être posée : les frontières nationales interviennent-elles dans la définition des composantes de la diversité interrégionale européenne, au point que les régions françaises se ressemblent plus entre elles qu'elles ne ressemblent aux autres régions de l'Europe communautaire ? Les processus de différenciation géographique sontils encore très sensibles aux limites territoriales des Etats?

Si l'on essaie d'évaluer ce qui, dans la diversité des régions européennes, revient à l'appartenance de ces régions à un même Etat, en mesurant la part de variation interrégionale d'un indicateur que ron peut attribuer à cette appartenance, plusieurs constatations s'imposent. Pour chacun des critères démographiques et socio-économiques pris en compte, les régions européennes appartenant à un même pays ont tendance à se ressembler plus entre elles qu'elles ne ressemblent aux autres régions de la Communauté. La diversité des situations nationales est le plus souvent à l'origine de la moitié, voire des deux-tiers de l'hétérogénéité des régions européennes (exemple : proportion des enfants 
dans la population totale ou encore taux de chómage des moins de 25 ans, produit intérieur brut par habitant, ...). Dans le cas où cette influence nationale apparaît relativement faible, c'est souvent près du quart de la différenciation régionale européenne qui s'explique par les écarts des situations nationales (exemple : taux de mortalité infantile ou taux de chômage).

Ces apparentes discontinuités aux frontières sont-elles le reflet d'interdépendances, de complémentarités entre les régions frontalières ? L'étude régionale permettra un examen appronfondi de ces liaisons dont l'intensité ne s'impose pas d'emblée. Si l'on considère par exemple les grandes infrastructures de liaison routière ou ferroviaire aux frontières, on voit immédiatement l'importance des liaisons entre Nord-Pas-de-Calais et Wallonie, et entre Alsace et régions allemandes voisines. Rien d'équivalent en Lorraine, et les grandes liaisons franco-italiennes sont peu nombreuses au regard de celles qui ouvrent I'Italie du Nord sur la Suisse, et au-delà sur l'Europe du Nord-Ouest. Vers l'Espagne, les grandes infrastructures routières paraissent davantage refléter une étroite liaison avec le Pays Basque et la Catalogne, mais y a-t-il régions transfrontalières pour autant?

Sait-on par ailleurs qu'on ne compte que 100.000 Français exerçant leur activité à l'étranger comme travailleurs frontaliers, essentiellement en Suisse (plus de $50 \%$ ) où ils travaillent surtout dans les services, ou en Allemagne (28\%), employés plutôt par l'industrie ? Les étrangers frontaliers venant travailler en France sont actuellement évalués à quelque 20.000 seulement, soit 13.000 originaires de Belgique, attirés surtout par les industries textiles de la région lilloise, et quelques milliers peut-être, en provenance d'Allemagne et d'Italie.

Le territoire français demeure un élément assez bien différencié de l'espace de l'Europe communautaire. II s'insère dans un ensemble où la dimension nationale reste une importante composante de la diversité géographique et de la forme des interactions spatiales qui s'y développent. 


\section{VERS DE NOUVELLES RÉGIONS}

\section{DÉBAT}

Animatrice : Thérèse SAINT-JULIEN

Avec : D. PUMAIN, V. REY, F. GAY et P.-Y. LE RHUN Compte rendu : M. CLARY

\section{Intervention de F. GAY : échelle inter et intra- régionale française} Etude de cas sur la Normandie éventuellement réunifiée.

Toutes les interventions qui ont précédé se situent au coeur du débat:

- la reconquête des rivages, des périphéries (Baudoin) peut s'appliquer à la Normandie,

- la région, espace d'identité avec priorité au culturel (Raffarin) : en Normandie, il y a un sentiment très fort en faveur de la petite région, du pays (Pays de Caux, Bessin), en revanche pas d'identité régionale au sens des régions actuelles, Haute et BasseNormandie,

- pas de contradiction entre gestion et démocratie, de nouvelles formes de démocratie sont à réinventer à chaque échelon géographique (Lévy) : la région doit être un espace de solidarité, la région ne va plus être qu'un espace de péréquation,

- rôle important du maillage urbain, l'importance du réseau des villes (Raffarin).

On peut appliquer toutes ces idées au cas de la Normandie.

On constate d'une part une permanence de cette région au cours des siècles depuis les ducs de Normandie et d'autre part un faible sentiment d'appartenance. Il y a permanence des limites que ce soit dans le projet d'A. Comte en 1848, celui de Vichy en 1941, celui de 1945, la première brèche s'observe avec J.-F. Gravier dans la proposition de 20 régions mais là aussi, le trait est plus fin entre la Haute et la Basse-Normandie. C'est seulement en 1956 que la séparation est faite.

II y a un sentiment d'unité et l'on peut constater la réalité de ce sentiment : le vote normand a bien existé en 1789, Louis XVI a été déclaré coupable mais non passible de la peine de mort. Siegfried en a tiré la formule, le Normand est un whig. Il y a un comportement normand. Mais ce sentiment d'unité est essentiellement donné de l'extérieur : une unité profonde existe, donnée par les abbayes, les plages, la fermette normande, "la grasse Normandie", le fleuve de lait et de beurre, toute une suite de stéréotypes.

Le sentiment d'appartenance est très faible peut-être dû à la très forte mobilité locale : record français des ZUP, déracinement des populations rurales qui vont travailler chez Citroën ou Renault, départ important des élites vers Paris (chaque jour, 3.500 cadres se dirigent vers Paris).

La division actuelle ne fait que renforcer les disparités qui existent : la Basse-Normandie risque de plus en plus de verser dans un grand Ouest dont elle sera la marge et la Haute-Normandie vers la mégapole de l'Europe du Nord Ouest mais elle sera là aussi 
sur les marges. A chaque fois, elle se situe sur les marges, la Basse-Seine devenant de plus en plus une région couloir où passent, sans s'arrêter hommes, marchandises, etc. Comment réconcilier maritimisation, souci de démocratie, dérive continentale... ?

La population se situe autour de 3 millions d'habitants, ce qui ne correspond pas à l'échelle européenne des régions. La Normandie se présente comme la première zone de faible résistance démographique à côté de l'Europe de l'Ouest (densité 100, Grande Bretagne 300 à 500 ). Le souci d'efficacité, de démocratie locale, de lutte contre le localisme, sont difficiles à intégrer. Le Pont de Normandie plus en aval que Tancarville, joindra le Havre à la Basse-Normandie. Il y aura des départements avec une gestion différente. On fait coïncider la société civile et le souci de responsabilité qui oblige à avoir un terrain de solidarité.

\section{Intervention de P.-Y. LE RHUN}

\section{Nantes et les Pays de la Loire - Projet}

On ne peut parler de Nantes et des Pays de Loire sans évoquer le problème d'une future région éventuelle sortant du cadre actuel (Bretagne et Grand-Ouest).

II n'y a plus à Nantes de discours de la défense des Pays de Loire depuis 2 à 3 ans, parallèlement à une convergence des grands partis pour une évolution vers le GrandOuest: notion encore très floue, à géométrie variable, au contenu indiscernable.

L'ambition de la bourgeoisie nantaise est d'ériger Nantes en capitale d'une grande région de l'Ouest. Dès 1941, A. DURAND, professeur d'économie politique, parlait de "Nantes dans la France de l'Ouest", démonstration non scientifique, aucune étude quantitative mais il occupait la position forte de premier adjoint au maire, il a donc écrit ce plaidoyer pour défendre Nantes dans la réforme régionale de Pétain, Président du Conseil Général après la guerre, jusqu'à 1973, sa position devient officielle.

Quelle région ? Dans l'Ouest, il n'y a que deux régions à stature européenne : la Normandie réunifiée et la Bretagne. Le problème est de savoir ce que sera cette future région sans la poussée des nécessités européennes : fusion Bretagne-Pays de Loire ou minorités rapprochées ? Le Grand-Ouest paraît mythique, excepté cette hypothèse. Une région qui s'étend vers l'Est suscite des réticences de Brest : nouvelle région BretagnePays de Loire à condition de ne pas négliger le capital Bretagne et son triple réseau de solidarité

- soutien des régions celtiques, des régions maritimes périphériques, des régions à culture minoritaire. Ce capital ne doit pas être dilué au nom de l'Europe.

- Une future région est au bout d'une dynamique qui reste à créer. Nantes a aujourd'hui la région qu'elle a méritée.

- Nous pouvons délimiter des espaces régionaux suivant des critères et aboutir à des régions géographiques mais nous ne pouvons pas prétendre passer du plan géographique au plan administratif. Nous n'avons pas la compétence à le faire, nous n'avons plus affaire à des agents économiques, mais à des citoyens dont nous devons analyser les besoins. 


\section{DISCUSSION}

\section{TH. SAINT-JULIEN}

A quoi doivent servir ces régions ? Qu'est-ce que la stature européenne ? Quelle volonté derrière l'agrandissement des régions ? Est-ce qu'on pense que des mailles plus grandes soient plus à l'échelle des mailles qui apparaissent ailleurs ? Est-ce l'échelle à laquelle se définissent aujourd'hui les dynamiques territoriales, socio-économiques ? Est-ce, au contraire, la meilleure échelle pour que des redistributions moins inégalitaires s'opèrent à l'intérieur de l'espace national ? Quels seront les principes qui présideront à un découpage ? Une grande part des dynamiques qui ont bouleversé la Normandie, la Bretagne, les Pays de Loire sont des processus qui se sont déployés à d'autres échelles géographiques, par exemple la distance à Paris est extrêmement importante de même que les tendances à la métropolisation continue ou la sélection entre grandes villes.

\section{F. GAY}

Une question fondamentale : la région pour faire quoi ?

Une nouvelle région au nom d'un projet : réunification de la Normandie pour un grand projet autour d'une tradition d'organisation, d'aménagement de l'espace, de projet territorial. La saga des Normands, c'est une conquête mais aussi une civilisation, la Grande Charte, la démocratie apportée en Grande Bretagne, le Domesday Book, le Grand Cadastre.

II s'agit d'une part de répondre à la mondialisation de l'économie et d'autre part d'agir localement.

\section{PHLIPPONEAU}

En 1955, régions de programmes, plan régional.

- L'espace-plan avec la première expérience de planification reste région de programme jusqu'en 1972, à la création des établissements publics régionaux. Seul rôle jusqu'en 1972 : analyser, émettre des avis sur la régionalisation des plans.

- A partir de 1972, le Plan Régional dispose de moyens financiers lui permettant de fournir des subventions aux départements.

- Lois de 1982-1983, la région devient un espace de gestion. Ne doit-elle pas servir à autre chose? 


\section{P.-Y. LE RHUN}

Dynamique à créer, pour faire quoi ? Face à la proposition implicite d'élaborer une grande région :

- position minimaliste région Bretagne-5 départements,

- possibilités d'évolution et pour cela débattre avec les voisines immédiates, discuter de façon démocratique.

\section{A. CHAUVET}

Le débat sur les nouvelles régions nous renvoie en l'An 900. Est-ce qu'on peut proposer un nouveau découpage ? Quels sont les nouveaux réseaux ? Axe Angers-Nantes-Paris ? Veut-on des régions en référence à une identité ancienne ou des régions réorganisées dans le cadre des réseaux urbains et des métropoles avec armature et dimension différentes?

\section{P. DUBOSCQ}

Le budget total de Bretagne-Normandie-Picardie équivaut à peu près au 1/100e du budget de la Bavière et Nous, géographes, nous parlons en superficie.

La première question n'est pas celle du découpage mais la région pour faire quoi ? Une région, comment a-t-elle fonctionné et pourquoi ? Est-ce que lorsqu'on parle de l'Europe des régions, on ne pourrait évoquer la réunification du pays basque ? Pourquoi pas des régions à l'échelle européenne?

\section{TH. BAUDOIN}

Face aux impératifs économiques et à la circulation internationale, les acteurs sont l'Etat et les villes. Or, les réalités culturelles reposent sur la certitude.

Le jacobinisme centralisateur a privé les villes de leur développement et brisé les identités culturelles. II nous reste, pour se dégager du jacobinisme, les impératifs économiques qui remettent en cause le découpage imposé par l'Etat : le Havre est le premier port français mais sous-préfecture, sa dimension économique n'est pas à l'échelle de l'Europe mais du Monde et les stratégies internationales prennent appui sur l'estuaire mais ni sur la Haute ni sur la Basse-Normandie : zone de conflit, de mouvance. 


\section{VERS DE NOUVELLES REG IONS}

\section{VANDENBROUCKE}

S'il y a des découpages, au niveau de l'Etat et des politiques:

- les régions auront un territoire, souci d'intervention, d'animation sur une dimension territoriale,

- capitales régionales de valeur européenne : par un souci de faire de Lyon une ville européenne. Pour qu'il y ait capitale de ce type-là, il est peut-être nécessaire d'avoir une région de dimension autre qu'aujourd'hui. Dans Rhône-Alpes, la capitale pourrait être Lyon-Grenoble, voire Saint-Etienne. Le rôle du géographe ? Montrer l'adéquation, la meilleure ou la moins mauvaise des solutions de découpage.

\section{J.-L. SMITH}

Le réseau de villes des années 70 et 80 n'est pas celui des années 30, celui de Christaller. C'est une seconde génération. II y a une nouvelle centralité interurbaine, un réseau social spatial et a-spatial. Il s'agit d'analyser les nouvelles formes de réseaux. Les déséquilibres ne sont pas à l'échelle régionale. L'Europe est un faux problème, c'est au niveau global, à l'échelle du Monde qu'il faut réfléchir. On entend un discours très nationaliste : la France en combien de régions ? Mais on ne s'est pas placé dans une perspective européenne : France des régions et non Europe des régions.

\section{F. DURAND-DASTÈS}

L'Europe est un problème et non un faux problème, elle existe.

- La régionalisation fonctionne autour de pôles, de réseaux, de flux qui déterminent des ensembles flous alors qu'en économie, les contraintes déterminent des ensembles non flous.

- Il est fascinant de voir à quel point une limite s'incruste facilement dans l'espace :

- 34 ans que les limites régionales durent

- l'Afrique est découpée (du net dans du flou)

-1956, réorganisation de l'espace indien

- les Etats des USA sont monstrueux et les limites tiennent.

II y a des moments privilégiés où se ferment des choses artificielles et qui durent, enracinées dans l'espace. 